\title{
Prevalence and epidemiology of canine and feline heartworm infection in Taiwan
}

\author{
Ta-Li Lu ${ }^{1,2}$, Jun-Yue Wong ${ }^{1,3}$, Ta-Lun Tan ${ }^{1,4}$ and Yong-Wei Hung ${ }^{1,2^{*}}$ \\ From 15th American Heartworm Society Triennial Symposium \\ New Orleans, LA, USA. September 11-13, 2016
}

\begin{abstract}
Background: Heartworm, Dirofilaria immitis, has long been recognized in Taiwanese dogs but feline heartworm infection has been largely overlooked by veterinarians and pet owners. The main goal of this study was to determine the prevalence and epidemiology of canine and feline heartworm infection in Taiwan.
\end{abstract}

Methods: Household dogs and cats were selected from 103 veterinary hospitals in 13 cities throughout Taiwan. All animals were at least 1 year old, had received no heartworm prevention for more than 1 year, and had lived in the same city for at least 1 year. Client consent was obtained and an owner questionnaire was completed for each patient. Blood samples were collected from each canine patient and tested at each veterinary hospital for microfilariae and for circulating antigen. A positive result on either test was considered to confirm mature heartworm infection. Blood was collected from each feline patient and examined for microfilariae and a feline heartworm antigen/antibody test was performed. Descriptive statistics were used for heartworm prevalence. Multivariate logistic regression analysis was used to determine the relationships between heartworm infection and multiple risk factors.

Results: A total of 2064 household dogs and 616 household cats from 103 veterinary hospitals throughout Taiwan were included in the study. The overall prevalence of canine heartworm disease was 22.8\% (471/2064). In heartworm-positive dogs, $63 \%$ were both microfilaria positive and antigen positive, 35\% were microfilaria negative and antigen positive, and only $2 \%$ were microfilaria positive and antigen negative. In the comparison of different life style groups, outdoor dogs $(N=797)$ had significantly higher heartworm prevalence rate than indoor dogs $(N=1267 ; p=0.000)$. The heartworm prevalence rate in dogs presented with dyspnea and cough was as high as $51 \%$. The overall prevalence of antibody-positive cats was $6.7 \%(41 / 616)$ and the antigen-positive prevalence rate was 3.1\% (19/616). In 41 antibody-positive cats, 6 of them were also antigen-positive. In 19 antigen-positive cats, 13 of them were antibody negative. In antibody-positive and antigen-negative cats, half had no clinical signs. In antigen-positive cats, 21\% had no clinical signs and only 38\% had classic heartworm clinical signs (dyspnea, cough, or gastrointestinal signs).

(Continued on next page)

\footnotetext{
* Correspondence: cardiospecial@gmail.com

${ }^{1}$ Taiwan Academy of Veterinary Internal Medicine, Taipei, Taiwan

${ }^{2}$ Cardiospecial Veterinary Hospital, No.34, Sec. 2, Heping E. Rd, Taipei 106 ,

Taiwan

Full list of author information is available at the end of the article
} 
(Continued from previous page)

Conclusions: Our canine study showed that southern and eastern Taiwan have the highest heartworm prevalence. Dogs not receiving preventive and living outdoors or those that have either cough or dyspnea have a high incidence of heartworm infection. We also confirmed that feline heartworm exposure exists in most cities in Taiwan. The diagnosis of feline heartworm infection will remain challenging for clinicians, however, without a consistent relationship between the presence of heartworm infection and clinical signs and the vagaries of microfilaria and antigen/antibody testing.

Keywords: Heartworm infection, Taiwan, Canine, Feline, Heartworm prevalence, Antigen testing, Antibody testing

\section{Background}

Heartworm, Dirofilaria immitis, is one of the most common and devastating parasitic disease in dogs. Taiwan, with its location in East Asia and its marine tropical climate, is a perfect environment for heartworm disease. Canine heartworm was first reported in Taiwan in 1935 [1]. There have been many reports of canine heartworm prevalence since then [2-11]. In 2001, Fan et al. investigated 523 pet dogs in Taipei and 141 dogs in mountain aboriginal districts and the heartworm prevalence was $13.8 \%(72 / 523)$ and $12.1 \%(17 / 141)$, respectively [12]. In 2003, a large scale study was published with 837 stray dogs and 1228 pet dogs from 13 cities throughout Taiwan. The overall heartworm prevalence rate in stray dogs and pet dogs was $57 \%$ and $26.5 \%$, respectively. The highest rate in pet dogs was found in Nantou (40.7\%) and lowest rate was in Hualien (4\%) [13]. None of these reports included the percentage of dogs on preventive. No large-scale studies on the prevalence of canine heartworm infection (HWI) in Taiwan have been published since then.

On the other hand, HWI in cats has been largely overlooked by veterinarians and owners. Compared with dogs, the diagnosis of feline HWI is more challenging. The first prevalence study of HWI in cats in Taipei city found only one antigen (Ag)-positive cat among 200 pet cats [14]. Recently, another study focusing on relationship between feline heartworm and lower airway signs revealed an antibody $(\mathrm{Ab})$ seroprevalence of $9.7 \%$ in 226 pet cats in Taipei city [15]. There have been no largescale studies of feline HWI prevalence involving different cities in Taiwan.

Many new heartworm diagnostic methods and preventive medications for both dogs and cats have been introduced in Taiwan in the last decade. We assume that those newer tools may affect the known prevalence and epidemiology of HWI in Taiwan and practitioners need updated information to educate clients and treat and prevent heartworm disease. This study is the first largescale study of feline heartworm disease prevalence in Taiwan and also the largest study of canine heartworm prevalence in pet dogs in Taiwan to date.
The goal of the study was to determine the current heartworm prevalence in pet dogs and cats in Taiwan and the potential risk factors that might have influence on heartworm prevalence.

\section{Methods}

Household dogs and cats were selected from 103 veterinary hospitals in 13 cities throughout Taiwan (Fig. 1). The number of hospitals involved in each city was decided based on the populations of pet dogs and cats. The target was evaluation of 30 dogs and 10 cats from each hospital. Dogs and cats included in this study were at least 1 year old, had received no heartworm prevention for more than 1 year, and had lived in the same city for at least 1 year. The study was approved by the ethical committee of Taiwan Academy of Veterinary Internal Medicine. For each patient, client consent was obtained and a questionnaire, including signalment, body weight, lifestyle (100\% of the time indoors, $100 \%$ of the time outdoors, $>50 \%$ indoors or $>50 \%$ outdoors), clinical signs at presentation [no clinical sign, dyspnea, cough, gastrointestinal (GI) sign or other clinical signs] was completed.

All blood samples were collected and tested at each veterinary hospital and the results were sent to the investigators using an online form. For each canine patient, at least $1 \mathrm{~mL}$ of blood was collected. The blood in EDTA tube was examined for circulating $\mathrm{Ag}(0.5 \mathrm{~mL})$ using commercial kit (SNAP ${ }^{\circ} 4 \mathrm{Dx}^{\circ}$ Plus, IDEXX Laboratories, Westbrook, Maine, USA) and for microfilariae $(0.5 \mathrm{~mL})$ using a direct blood smear technique by placing $0.5 \mathrm{~mL}$ blood sample from EDTA tube on the slide with cover slips over top and examination under the microscope. Either Ag or microfilariae positive was considered to confirm HWI.

For each feline patient, at least $2.5 \mathrm{~mL}$ of blood was collected in EDTA tubes and was examined for microfilariae $(0.5 \mathrm{~mL})$, also using a direct blood smear technique. Serum was collected from the nonanticoagulant tube and the feline heartworm Ab test was done using a commercial kit (Solo Step ${ }^{\circ} \mathrm{FH}$, Heska, Loveland, Colorado, USA) and the Ag test was 

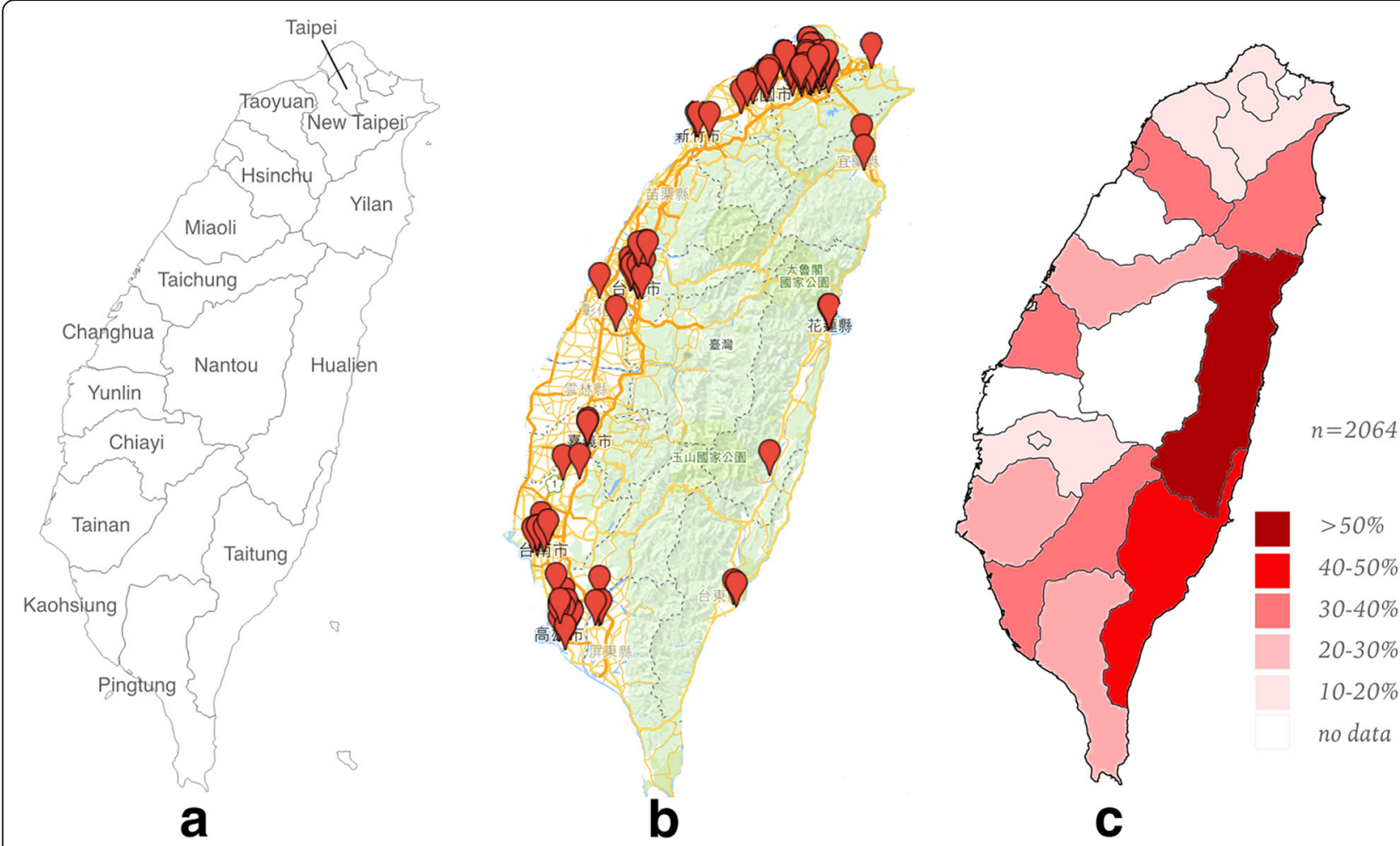

Fig. 1 a Map of Taiwan. b Study sampling locations. c Canine heartworm prevalence in Taiwan

performed using a commercial kit (SNAP $^{\circ}$ Feline Heartworm, IDEXX Laboratories; Witness ${ }^{\circ}$ HW, Zoetis, Parsippany, New Jersey, USA).

Descriptive statistics were used to demonstrate heartworm prevalence. Prevalence results are expressed as median (range). Multivariate logistic regression analysis was used to determine the relationships between heartworm prevalence and the presence of multiple risk factors obtained from the questionnaire.

\section{Results}

\section{Dogs}

In the canine study, 2064 pet dogs were included and tested for heartworm disease from April to October 2015. There were 1071 male and 993 female dogs, with a median age of 7 years (range from 1 to 19 years old) and median body weight of $8.3 \mathrm{~kg}$ (range from 1 to $60 \mathrm{~kg}$ ). The pet dogs included 655 mixed-breed dogs, 253 Maltese, 221 Miniature Poodles, 121 Dachshunds, 120 Miniature Schnauzers, 102 Chihuahuas, 57 Golden Retrievers, 45 Beagles, 42 Labrador retrievers, 38 Shih-Tzus, 37 Pomeranians, 28 Spitzs, and 345 dogs representing other breeds.

The overall prevalence rate of canine heartworm disease was $22.8 \%$, ranging from $12.1 \%$ to $51.1 \%$ in different cities (Table 1). Hualien had highest prevalence rate of $51.1 \%$ (44/86), followed by Taitung $(46.7 \%, 14 / 30)$ and
Yilan $(36.36 \%, 12 / 33)$. Taipei had lowest prevalence rate of $12.1 \%$ (44/365), followed by New Taipei City (13.6\%, $55 / 406)$ and Chiayi $(13.9 \%, 5 / 36)$. Overall, eastern and southern Taiwan had higher prevalence rates than northern Taiwan (Fig. 1).

Table 1 Prevalence of canine heartworm disease in different cities in Taiwan

\begin{tabular}{llll}
\hline Area & $\begin{array}{l}\text { Sample } \\
\text { Number }\end{array}$ & Heartworm (+) Number & Heartworm (+) \% \\
\hline Hualien & 86 & 44 & $51.16 \%$ \\
Taitung & 30 & 14 & $46.67 \%$ \\
Yilan & 33 & 12 & $36.36 \%$ \\
Hsinchu & 44 & 15 & $34.09 \%$ \\
Kaohsiung & 316 & 104 & $32.91 \%$ \\
Changhua & 44 & 14 & $31.82 \%$ \\
Taichung & 280 & 70 & $25.00 \%$ \\
Tainan & 237 & 59 & $24.89 \%$ \\
Pingtung & 35 & 7 & $20.00 \%$ \\
Taoyuan & 152 & 28 & $18.42 \%$ \\
Chiayi & 36 & 5 & $13.89 \%$ \\
New Taipei & 406 & 55 & $13.55 \%$ \\
Taipei & 365 & 44 & $12.05 \%$ \\
Total & 2064 & 471 & $22.82 \%$ \\
\hline
\end{tabular}


Among all dogs, 28\% (575/2064) lived 100\% indoors, $33 \%(692 / 2064)$ lived $>50 \%$ indoors, $17 \%(362 / 2064)$ lived $>50 \%$ outdoors, and 21\% (435/2064) lived 100\% outdoors. Comparing the different lifestyle groups, the $100 \%$ outdoor group had the highest prevalence rate of $55.6 \%$, followed by $32.3 \%$ of the $>50 \%$ outdoor group, $10 \%$ of the $>50 \%$ indoor group, and $7.5 \%$ of the $100 \%$ indoor group (Fig. 2).

The prevalence difference between each lifestyle group was also noted in each city. For example, in Taipei, which had lowest prevalence rate in Taiwan, canine HWIs were still frequently observed in the $100 \%$ outdoor group with prevalence rate of $53 \%(16 / 30)$, followed by $21 \%(15 / 70)$ of the $>50 \%$ outdoor group, $7 \%(10 / 143)$ of the $>50 \%$ indoor group, and $2 \%(3 / 122)$ of the $100 \%$ indoor group. This high frequency of indoor housing resulted in $<13 \%$ of dogs being Ag-positive in Taipei. In Hualien, the 100\% outdoor group had highest prevalence rate of $70 \%$ (35/50), followed by $40 \%(2 / 5)$ of the $>50 \%$ outdoor group, $31 \%$ (4/ 13 ) of the $>50 \%$ indoor group, and $17 \%(3 / 18)$ of the $100 \%$ indoor group. The high frequency of outdoor housing resulted in $>50 \%$ of dogs being Ag-positive in Hualien. In heartworm-positive dogs, $63 \%$ were both microfilaria positive and $\mathrm{Ag}$ positive, $35 \%$ were microfilaria negative and Ag positive, and only $2 \%$ were microfilaria positive and Ag negative.

Of all the dogs included in this study, 55\% (1125/ 2064) presented without clinical signs, $14 \%(282 / 2064)$ had cough, 6\% (120/2064) had dyspnea, 6\% (132/2064) had gastrointestinal signs, and 20\% (405/2064) had other clinical signs. In heartworm-positive dogs, 34\% (159/ 471) presented without clinical signs, $28 \%(133 / 471)$ had cough, 13\% (61/471) had dyspnea, 4\% (18/471) had GI signs, and 21\% (100/471) had other clinical signs. On the other hand, in dogs presented without clinical signs, 14\% (159/1125) were heartworm positive. The heartworm prevalence rate in dogs presented with cough was $47 \%(133 / 282)$ and dogs presented with dyspnea was $51 \%(61 / 120)$.

Multiple logistic regression results for risk factors of canine HWI are shown in Table 2. We found that spending $>50 \%$ of time outdoors and having cough or dyspnea were independent risk factors for canine HWI.

\section{Cats}

In the feline study, 616 pet cats were tested for HWI from April to October 2015. There were 327 male and 289 female cats, with a median age of 6 years (range 1 to 20 years) and median body weight of $4 \mathrm{~kg}$ (range 1.5 to $9.5 \mathrm{~kg}$ ). We included 415 Domestic shorthair, 69 Persian, 56 Chinchilla, 30 American Shorthair, 15 Scottish Fold, 8 British Shorthair, 5 Himalayan, 4 Bengal, 3 Ragdoll, 3 Russian Blue, 2 Japanese Botail, 2 Abyssinian, 2 Exotic Shorthair, 1 American Curl, and 1 Siamese.

The overall Ab-positive rate was 6.7\%, ranging from $0 \%$ to $10.3 \%$ in different cities. (Table 3 ) The city of Chiayi had highest Ab-positive rate (10.3\%, 3/29), followed by Taipei $(9.2 \%, 14 / 152)$ and Taichung $(8.8 \%, 6 /$ 68). There were four cities (Changhua, $n=11$ cats;

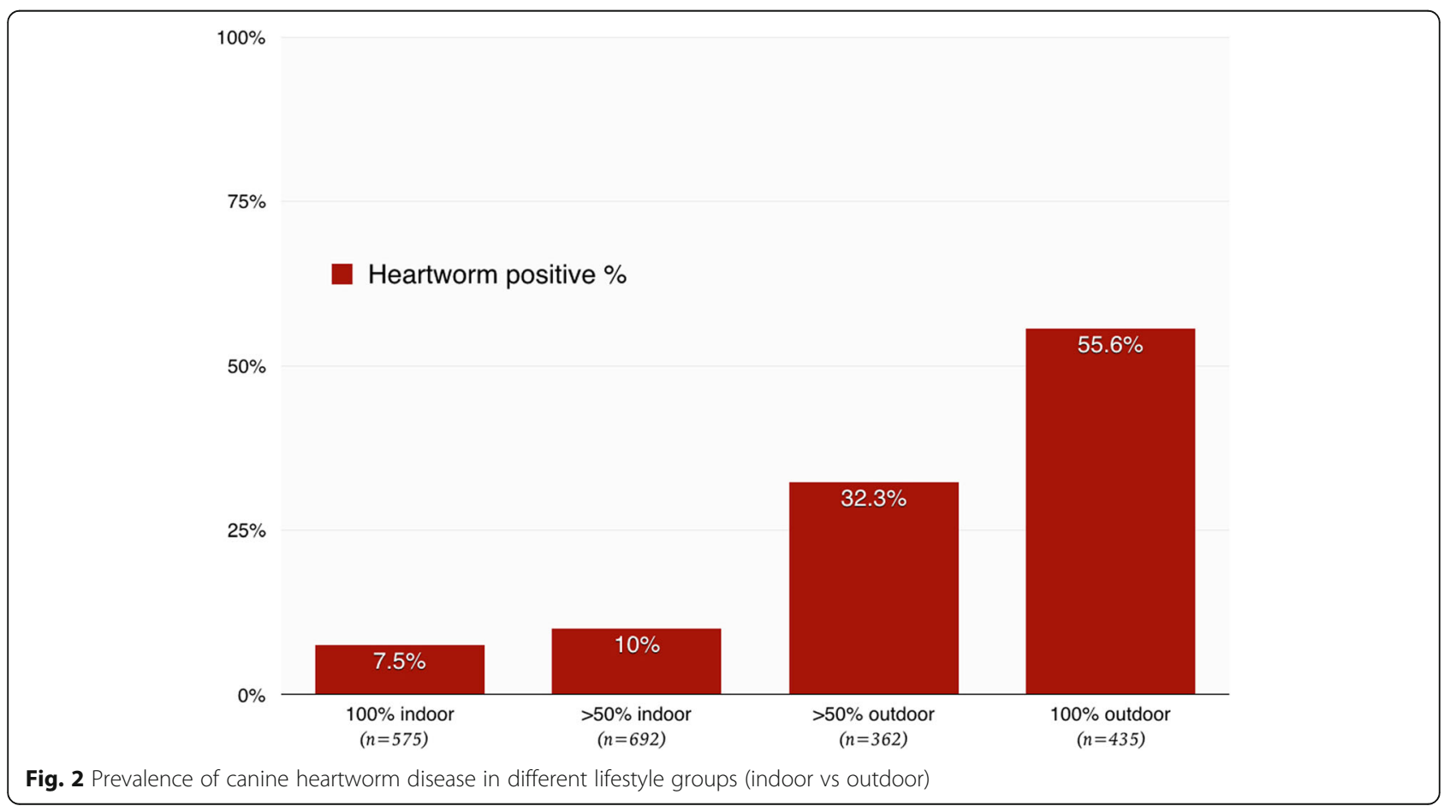


Table 2 Results of multiple logistic regression on risk factors for canine heartworm disease

\begin{tabular}{|c|c|c|c|c|c|c|}
\hline & & Regression Coefficient (B) & Standard Error (SE) & $P$ value & Odds Ratio (OR) & 95\% Confidence Interval (Cl) \\
\hline \multirow[t]{4}{*}{ Lifestyle } & $100 \%$ indoor & reference & & & & \\
\hline & $>50 \%$ indoor & 0.161 & 0.209 & 0.441 & 1.174 & $0.780-1.768$ \\
\hline & $>50 \%$ outdoor & 1.700 & 0.201 & $0.000^{*}$ & 5.475 & $3.694-8.115$ \\
\hline & $100 \%$ outdoor & 2.591 & 0.193 & $0.000^{*}$ & 13.340 & $9.142-19.464$ \\
\hline \multirow[t]{2}{*}{ Dyspnea } & No & reference & & & & \\
\hline & Yes & 1.370 & 0.233 & $0.000^{*}$ & 3.934 & $2.493-6.208$ \\
\hline \multirow[t]{2}{*}{ Gl signs } & No & reference & & & & \\
\hline & Yes & -0.265 & 0.288 & 0.358 & 0.767 & $0.436-1.350$ \\
\hline \multirow[t]{2}{*}{ Cough } & No & reference & & & & \\
\hline & Yes & 1.657 & 0.167 & $0.000^{*}$ & 5.242 & $3.779-7.273$ \\
\hline \multirow[t]{2}{*}{ Other clinical signs } & No & reference & & & & \\
\hline & Yes & 0.415 & 0.160 & $0.009^{*}$ & 1.515 & $1.108-2.071$ \\
\hline
\end{tabular}

*Significant

Hsinchu, $n=8$; Pingtung, $n=7$; Taitung, $n=6$ ) where no Ab-positive cases were reported.

The overall Ag-positive rate was 3.1\%, ranging from $0 \%$ to $9.5 \%$ in different cities. (Table 3 ) The city of Kaohsiung had highest Ag-positive rate (9.5\%, 8/84), followed by Taipei $(4.6 \%, 7 / 152)$ and Taichung $(2.9 \%, 2 /$ 68). There were eight cities (Taoyuan, $n=41$ cats; Chiayi, $n=29$; Hualien, $n=21$; Yilan, $n=13$; Changhua, $\mathrm{n}=11$; Hsinchu, $\mathrm{n}=8$; Pingtung, $\mathrm{n}=7$; Taitung, $\mathrm{n}=6$ ) where no Ag-positive cats were detected.

Among all cats included in this study, 64\% (396/616) lived $100 \%$ indoors, $13 \%(79 / 616)$ lived $>50 \%$ indoors, $7 \%(44 / 616)$ lived $>50 \%$ outdoors and $16 \%$ (97/616) lived

Table 3 Prevalence of feline heartworm antigen (Ag) and antibody (Ab) seropositivity in different cities in Taiwan

\begin{tabular}{|c|c|c|c|c|c|c|c|}
\hline \multirow[t]{2}{*}{ Area } & \multirow{2}{*}{$\begin{array}{l}\text { Sample } \\
\text { number }\end{array}$} & \multicolumn{2}{|c|}{$\mathrm{Ag}(+)$} & \multicolumn{2}{|c|}{$\mathrm{Ab}(+)$} & \multicolumn{2}{|c|}{$M f(+)$} \\
\hline & & (n) & (\%) & (n) & $(\%)$ & $(n)$ & $(\%)$ \\
\hline Kaohsiung & 84 & 8 & $9.5 \%$ & 5 & $6.0 \%$ & 2 & $2.4 \%$ \\
\hline Taipei & 152 & 7 & $4.6 \%$ & 14 & $9.2 \%$ & 1 & $0.7 \%$ \\
\hline Taichung & 68 & 2 & $2.9 \%$ & 6 & $8.8 \%$ & 0 & $0.0 \%$ \\
\hline Tainan & 60 & 1 & $1.7 \%$ & 5 & $8.3 \%$ & 0 & $0.0 \%$ \\
\hline New Taipei & 116 & 1 & $0.9 \%$ & 5 & $4.3 \%$ & 0 & $0.0 \%$ \\
\hline Chiayi & 29 & 0 & $0.0 \%$ & 3 & $10.3 \%$ & 1 & $3.4 \%$ \\
\hline Yilan & 13 & 0 & $0.0 \%$ & 1 & $7.7 \%$ & 0 & $0.0 \%$ \\
\hline Hualien & 21 & 0 & $0.0 \%$ & 1 & $4.8 \%$ & 0 & $0.0 \%$ \\
\hline Taoyuan & 41 & 0 & $0.0 \%$ & 1 & $2.4 \%$ & 0 & $0.0 \%$ \\
\hline Changhua & 11 & 0 & $0.0 \%$ & 0 & $0.0 \%$ & 0 & $0.0 \%$ \\
\hline Hsinchu & 8 & 0 & $0.0 \%$ & 0 & $0.0 \%$ & 0 & $0.0 \%$ \\
\hline Pingtung & 7 & 0 & $0.0 \%$ & 0 & $0.0 \%$ & 0 & $0.0 \%$ \\
\hline Taitung & 6 & 0 & $0.0 \%$ & 0 & $0.0 \%$ & 0 & $0.0 \%$ \\
\hline Total & 616 & 19 & $3.1 \%$ & 41 & $6.7 \%$ & 4 & $0.6 \%$ \\
\hline
\end{tabular}

Ag Antigen test, $A b$ Antibody test, Mf Microfilaria test, (+) positive result
$100 \%$ outdoors. There was no heartworm prevalence difference among each lifestyle group.

Fifteen percent (6/41) of Ab-positive cats were also Ag positive. Sixty-eight percent (13/19) of Ag-positive cats were $\mathrm{Ab}$ negative. Microfilariemia was found in four cats, two of which were both $\mathrm{Ab}$ and $\mathrm{Ag}$ positive while the other two were $\mathrm{Ab}$ positive but Ag negative.

For all cats included in this study, 55\% (336/616) of presented without clinical signs, 11\% (65/616) had cough, 6\% (34/616) had dyspnea, 6\% (36/616) had GI signs, and $24 \%(145 / 616)$ had other clinical signs. In Abpositive, but Ag-negative cats, 51\% (18/35) had no clinical signs, 11\% (4/35) had cough, 6\% (2/35) had GI signs, none had dyspnea, and 31\% (11/35) had other clinical signs. In Ag-positive cats, 22\% (4/19) had no clinical signs, 11\% (2/19) had dyspnea, 11\% (3/19) had cough, $11 \%(2 / 19)$ had gastrointestinal signs, and 44\% (8/19) had other clinical signs.

Multiple logistic regression results for risk factors of feline heartworm Ab seropositivity are shown in Table 4. There was no significant correlation among clinical signs, lifestyle, or Ab seropositivity.

\section{Discussion}

\section{Canine heartworm infection}

In the canine study, the overall heartworm prevalence rate (Table 1 ) in Taiwanese pet dogs was $22.8 \%$, which is slightly lower than a previous report of $26.5 \%$ [13]. In Hsinchu and Tainan, the prevalence was slightly decreased, but in other southern and eastern cities (Kaohsiung, Taitung, Yilan, Pingtung), the prevalence rate has increased dramatically. The biggest change was in Hualien. In our study, Hualien had the highest rate of HWI, $51.1 \%$, but in Wu \& Fan's earlier study, Hualien had lowest rate of $4 \%$. [13] In central Taiwan cities (Taichung, Changhua), the prevalence rate of our study was higher 
Table 4 Results of multiple logistic regression on risk factors for feline heartworm antibody (Ab) seropositivity

\begin{tabular}{|c|c|c|c|c|c|c|}
\hline & & Regression coefficient (B) & Standard Error (SE) & $P$ value & Odds Ratio $(\mathrm{O}) \mathrm{R}$ & 95\% Confidence Interval (Cl) \\
\hline \multirow[t]{4}{*}{ Lifestyle } & $100 \%$ indoor & reference & & 0.672 & & \\
\hline & $>50 \%$ indoor & -0.772 & 0.628 & 0.219 & 0.462 & $0.135-1.583$ \\
\hline & $>50 \%$ outdoor & -0.175 & 0.640 & 0.784 & 0.839 & $0.240-2.940$ \\
\hline & $100 \%$ outdoor & -0.037 & 0.449 & 0.934 & 0.963 & $0.399-2.324$ \\
\hline \multirow[t]{2}{*}{ Dyspnea } & No & reference & & & & \\
\hline & Yes & -0.553 & 1.046 & 0.597 & 0.575 & $0.074-4.464$ \\
\hline \multirow[t]{2}{*}{ Gl signs } & No & reference & & & & \\
\hline & Yes & 0.058 & 0.770 & 0.940 & 1.059 & $0.234-4.789$ \\
\hline \multirow[t]{2}{*}{ Cough } & No & reference & & & & \\
\hline & Yes & 0.602 & 0.495 & 0.224 & 1.825 & $0.692-4.8181$ \\
\hline \multirow[t]{2}{*}{ Other clinical signs } & No & reference & & & & \\
\hline & Yes & 0.712 & 0.380 & 0.061 & 2.039 & $0.968-4.296$ \\
\hline
\end{tabular}

than previous reported [16]. In Taipei city, the current prevalence rate was slightly lower than that seen in a previous study [12]. These comparisons suggest that even though the overall prevalence rate was slightly decreased, which may be attributed to the decrease in prevalence in northern Taiwan, canine heartworm has become more prevalent in recent years in eastern, central, and southern Taiwan. Our canine heartworm prevalence rate was similar to earlier studies in adjacent countries, including China, Korea, and Japan [17-19].

Many factors can influence heartworm infection prevalence rates, including animal selection (pet dogs or stray dogs, on prevention or not on prevention), diagnostic test selection (ELISA Ag test, microfilaria test, or PCR), and climate conditions over the previous several years. The present study was designed to match the clinical experience, reflective of what veterinarians actually see, with all subjects being actual hospital patients. This more representative population in our study allows our data to be used by Taiwanese veterinarians as a tool for diagnostic decision making and client education.

An outdoor lifestyle was one of the major risk factors for canine heartworm disease, likely because of increased mosquito exposure in outdoor environments [20]. Our study indicated that, compared with $100 \%$ indoor dogs, those spending more than $50 \%$ of the time outdoors were 5.5 times more likely to contract HWI; for $100 \%$ outdoor dogs, the infection rate was 13.4 times higher. There was no significant difference in heartworm prevalence rate between $100 \%$ indoor dogs and dogs spending less than $50 \%$ of time outdoors (Table 2). On the other hand, we found that $100 \%$ indoor housing did not completely protect the dogs from HWI. There were 43 heartworm-positive dogs living $100 \%$ indoors $(9.1 \%$ of all HWI cases).

The variation in the canine heartworm prevalence rate among lifestyle groups was found in every city studied.
We also found that the overall prevalence rate in each city was largely related to the distribution of lifestyle group. Higher outdoor housing frequency result in higher overall prevalence of HWI. The pet dogs' indoor/ outdoor housing frequency difference between cities may due to human population density and housing variability. For example, Taipei, the biggest city in Taiwan, has the highest population density, with most people live in small apartments without yards or balconies. Therefore, most pet dogs live indoors. To the contrary, Hualien has lower population density and most people live in townhouses with yards, so there is space for pet dogs to more readily spend time outside of the house.

In conclusion, the overall prevalence rate in each city appears to be influenced by multiple factors. We suggest that a finding of lower heartworm prevalence does not necessarily indicate lower regional risk of potential HWI, as mosquito exposure plays an important role. Therefore, when assessing risk of HWI, lifestyle of pet and owner must also be considered.

We followed American Heartworm Society (AHS) guidelines to diagnose canine heartworm disease with both $\mathrm{Ag}$ and microfilaria tests. False-negative Ag test results may occur when the worms are still immature, worm burden is very low, in all-male infections, or with the formation of immune-complexes [21]. Compared with the $\mathrm{Ag}$ test, the microfilaria test has lower sensitivity, with $20 \%$ of heartworm-infected dogs not being microfilaremic [21]. Microfilaria testing can be performed using direct blood smear, buffy coat smear, millipore filtration, or the modified Knott test [22]. The latter is suggested in the AHS guidelines, but since not all hospitals in this study had the ability or equipment to do the modified Knott test, we chose the direct blood smear method with $0.5 \mathrm{~mL}$ blood. In our study, $63 \%$ (295/471) of heartworm-positive dogs were both microfilaria and Ag positive, and 35\% (166/471) of them were 
Ag positive but microfilaria negative. False-negative microfilaria test results were more common than described in the AHS guidelines, probably reflecting the methodology used in study. There were 10 Ag-negative cases that had microfilaria in the blood, supporting the AHS Guidelines' recommendation to perform both microfilaria and Ag tests on every patient.

Clinical signs of heartworm-infected dog are mostly respiratory signs. In mild or early infections, cough alone may be noted. In more severe and chronic cases, dyspnea, exercise intolerance, or right-side heart failure signs may be present [21]. In this study, 34\% (159/471) of heartworm-positive dogs were asymptomatic. Cough $(28 \%, 133 / 471)$ and dyspnea $(13 \%, 61 / 471)$ were the two most frequently seen clinical signs in heartwormpositive dogs. On the other hand, heartworm prevalence rates in dogs presented with cough $(n=282)$ and dyspnea $(n=120)$ were $47 \%$ and $51 \%$, respectively. Statistical analysis also indicated that having cough or dyspnea was associated with an increased risk of HWI. When analyzing cough and dyspnea signs in different lifestyle groups, for dogs with cough or dyspnea that lived more than $50 \%$ of time outdoors, the HWI prevalence rate was increased to $73 \%$. For dogs with cough or dyspnea spending less than $50 \%$ of time outdoors, the HWI prevalence rate was decreased to $15 \%$ to $24 \%$. These results indicate that unprotected outdoor dogs presenting with cough or dyspnea were at the highest risk for HWI.

\section{Feline heartworm infection}

Feline HWI is very challenging to veterinarians for several reasons. One reason is that in addition to the lung damage caused by adult heartworms, immature worms can cause heartworm-associated respiratory disease (HARD) without a mature infection resulting [23, 24]. Antigen testing is used to detect adult worm Ag. An Agpositive result confirms the presence of mature female heartworm(s). Antibody testing is used to detect antibodies formed in response to heartworm larvae. An Abpositive result confirms that the cat is at risk, and that detected antibodies may indicate a mature infection, ongoing exposure to heartworm larvae, or previous exposure [25]. False-negative Ag test results may be due to low worm burden, only male worm infection, immature worms or $\mathrm{Ag}-\mathrm{Ab}$ complexes [26, 27]. False-negative $\mathrm{Ab}$ test results may be due to an inadequate $\mathrm{Ab}$ response after exposure or pre-patent infections ( $<3$ month) [15, 28]. A negative $\mathrm{Ag}$ or $\mathrm{Ab}$ test result cannot completely rule out the HWI or exposure [24, 29].

In the feline study, our Ag prevalence rate in Taipei was higher than previously reported [14], which may indicate an increasing prevalence of feline heartworm disease in the past 11 years. The $\mathrm{Ab}$ prevalence rate in
Taipei, however, was lower than that found in a previous study [15]. This difference may be due to our study having fewer cats with lower airway clinical signs (18\%) than in the previous study (45\%). Compared with other Asian reports, our Ag prevalence rate (3.1\%) was similar to those in Japan (3\%) and Korea (2.6\%) [30, 31]. Our Ab prevalence rate $(6.7 \%)$ was lower than report from the United States (12\%) [32]. Our study detected Ab-positive cats in every city except Changhua, Hsinchu, Pingtung, and Taitung. Each of these cites had less than 12 cases (Changhua, $n=11$; Hsinchu, $n=8$; Pingtung, $n=7$; Taitung, $n=6$ ), which may be the reason that no infected or Ab-positive cats were detected. Based on the rest of prevalence data in Taiwan, we confirmed that heartworm exposure in pet cats is widely seen in Taiwan and we believe that all cats are at risk for heartworm exposure. Year-round heartworm prevention for cats in Taiwan is suggested by our study results, although further and larger studies are needed to fully understand the epidemiology of heartworms in cats in Taiwan.

While most studies have suggested that outdoor exposure was a risk factor for feline heartworm exposure $[15,25,33]$, one study found no correlation between outdoor exposure time and heartworm exposure [34]. In the current study, we also did not show correlation between outdoor exposure and heartworm exposure, but, importantly, we found, as did previous studies, that $100 \%$ indoor cats were still at risk for heartworm exposure. Heartworm prevention is suggested for all pet cats, regardless of lifestyle.

Fifteen percent (6/41) of our Ab-positive cats were also Ag positive, which indicated they were not only being exposed to heartworm but also had at least one adult female heartworm. All six of these cats were presented with clinical signs (two with cough, one with dyspnea, three with other clinical signs). Sixty-eight (13/19) of our $\mathrm{Ag}$-positive cats were $\mathrm{Ab}$ negative. Of these 13 cats, 4 had no clinical signs and 9 had clinical signs (one with cough, one with dyspnea, two with GI signs, five with other clinical signs). The finding of cats that were $\mathrm{Ag}$ positive but $\mathrm{Ab}$ negative may be due to failure to produce detectable antibodies in infected cats [28], or low sensitivity of the Ab test [29], or simply the small numbers of Ag-positive cats.

Previous studies have shown $10 \%$ to $20 \%$ of cats with proven HWI to be Ab negative [25]. Some authors advocate using the $\mathrm{Ab}$ test as a screening test for feline HWI, then using the Ag test as a confirmation test when a positive $\mathrm{Ab}$ test result is found [25, 33]. Because we found a high incidence of falsenegative $\mathrm{Ab}$ test results in Ag-positive cats, we suggest when screening for HWI in cats, $\mathrm{Ag}$ and $\mathrm{Ab}$ tests should be done together to get higher diagnostic sensitivity $[23,24,26]$. 
Clinical signs of feline heartworm disease are more complicated than canine heartworm disease [25]. With each different infection stage, the infected cat may show mild to severe, chronic to acute clinical signs. Respiratory signs are most common. Neurologic and GI signs may also be noted [24]. In this study, $44 \%$ of Ab-positive cats presented without clinical signs, $15 \%$ had cough, 5\% had GI signs, $2 \%$ had dyspnea, and 34\% had other clinical signs. In Ag-positive cats, $21 \%$ had no clinical signs, $16 \%$ had cough, $11 \%$ had dyspnea, $11 \%$ had GI signs, and $42 \%$ had other clinical signs. Therefore, only $39 \%$ of Ag-positive cats presented with the most common heartworm clinical signs (cough, dyspnea, GI signs), making the diagnosis of heartworm in cats a more challenging task. The presence of cough and dyspnea did not have significant correlation to heartworm exposure in our study, similar to a previous report [15]. There was no significant difference between $\mathrm{Ab}$ and $\mathrm{Ag}$ positive rates between the group with cough or dyspnea and the group with no cough or dyspnea.

Our study had some limitations. First, case numbers in some cities were not large enough to ensure that they accurately represented regional prevalence. This may have resulted in over- or underestimation of prevalence rates in certain cities, with specific concerns that feline heartworm prevalence underestimation may have occurred in sites where we detected neither Ag- nor Abpositive cases. Small numbers of cats in some areas make firm conclusions difficult. Second, our study did not have necropsy confirmation. A false-negative or false-positive $\mathrm{Ag}$ and $\mathrm{Ab}$ result may cause underestimation or overestimation of the heartworm prevalence rate in both dogs and cats. This is more often of concern in cats [29]. Third, because all patients in our study were pet dogs and cats from veterinary hospitals, our study does not answer the question of heartworm prevalence in stray dogs or owned dogs and cats not receiving veterinary care. Fourth, the heat treatment technique was not used on negative Ag samples, which would likely have increased canine results by approximately $7 \%$ and feline results by even more.

\section{Conclusions}

The overall prevalence rate of heartworm disease in unprotected pet dogs in Taiwan was $22.8 \%$. Southern and eastern Taiwan had higher prevalence rates than northern Taiwan. Pet dogs that spent more than $50 \%$ of their time outdoors that were presented with cough or dyspnea had a higher risk for heartworm disease. We also confirmed that feline heartworm exposure exists in most parts of Taiwan, with an overall Ab prevalence rate of 6.7\% and an Ag prevalence rate of 3.1\%. Lifestyle had no statistically significant impact on heartworm exposure in pet cats, including the important fact that living indoors did not fully protect pet cats from heartworm exposure. Based on our study result, we suggest that heartworm prevention should be administered to all pet dogs and cats in Taiwan.

\section{Additional file}

Additional file 1: Participating Veterinary Hospitals. (DOCX 13 kb)

\section{Abbreviations}

Ab: Antibody; Ag: Antigen; AHS: American heartworm society;

ELISA: Enzyme-linked immunosorbent assay; Gl: Gastrointestinal;

HARD: Heartworm-associated respiratory disease; HWI: Heartworm infection

\section{Acknowledgements}

The authors are sincerely grateful to all of the veterinary hospitals and veterinarians that joined this study, and all mentors, especially Dr. Clarke E. Atkins, who inspired and guided us in this study from the beginning to the end. Without their effort and help, this study could not have been done. A list of participating veterinary hospitals is available in Additional file 1.

\section{Funding}

This study was funded by Taiwan Academy of Veterinary Internal Medicine, Merial, and Zoetis. Canine and feline heartworm antigen test kit were sponsored by IDEXX Laboratories. The article's publication fee was funded by American Heartworm Society.

\section{Availability of data and materials}

All the data obtained during this study and supporting the conclusions of this article are included in the article.

\section{About this supplement}

This article has been published as part of Parasites and Vectors Volume 10 Supplement 2, 2017: Proceedings of the 15th American Heartworm Society Triennial Symposium 2016. The full contents of the supplement are available online at https://parasitesandvectors.biomedcentral.com/articles/ supplements/volume-10-supplement-2.

\section{Authors' contributions}

TLL designed the study, collected data from each participating hospital, analyzed the data, and wrote the manuscript. YWH supervised all stages of the work, including designing the study and revising the manuscript. BYW and DT participated in the study coordination, veterinary hospital recruitment, funding acquisition, and revising the manuscript. All authors read and approved the final version of the manuscript.

\section{Ethics approval and consent to participate}

The study was approved by the ethical committee of Taiwan Academy of Veterinary Internal Medicine. Client consent was obtained before entering this study for each patient.

\section{Competing interests}

TLL received travel funding and speaking fees from IDEXX Laboratories in the last 2 years. Other authors declare that they have no competing interests.

\section{Publisher's Note}

Springer Nature remains neutral with regard to jurisdictional claims in published maps and institutional affiliations.

\section{Author details}

${ }^{1}$ Taiwan Academy of Veterinary Internal Medicine, Taipei, Taiwan.

${ }^{2}$ Cardiospecial Veterinary Hospital, No.34, Sec. 2, Heping E. Rd, Taipei 106, Taiwan. ${ }^{3}$ Cambridge Animal Hospital, Taipei, Taiwan. ${ }^{4}$ Manhattan Veterinary Hospital, Taipei, Taiwan. 


\section{Published: 9 November 2017}

\section{References}

1. Miyamoto S, Uchida CH. Uber Filaria immitis bei Hunden in Formosa. J Formos Med Assoc. 1935;34:448-51.

2. Hsieh $\mathrm{HC}$, Chuang $\mathrm{CH}$. A note on the periodic appearance of microfilaria of Dirofilaria immitis Leidy in the peripheral blood of an indigenous dog in Taiwan (Formosa). J Formos Med Assoc. 1956;55:502-4.

3. Huang WH, Chiu JK, Kao CT. A survey of the endoparasite fauna of stray dogs in North Taiwan. J Formos Med Assoc. 1957;56:614 (in Chinese).

4. Yang C, Hsieh KL. Survey and treatment of Dirofilaria immitis infection among the dogs in Central Taiwan. Taiwan J Vet Med Ani Husb. 1987;50: 113-7 (in Chinese).

5. Wu CC, Fan PC, Lin CY. Dirofilaria immitis infection among stray dogs used in the laboratory. Chin J Parasitol. 1992:5:61-5.

6 Tin $\mathrm{CH}$, Wang JS. Investigation of Dirofilaria immitis infections of stray dogs in both cities of Taichung and Keelung. Chin J Parasitol. 1994;7:24.

7 Liang SL, Huang HP, Yang HL, Wong SS, Chen KY, Fei CY. Prevalence of canine dirofilariasis in pet dogs from Taipei area. J Chin Soc Vet Sci. 1996;22 167-74 (in Chinese).

8 Wang LC. Canine filarial infections in north Taiwan. Acta Trop. 1997;68: 115-20.

9 Fang BS, Lin JC, Chen PC, Lin WL. Short report: survey of dog heartworm in stray dogs in Kaohsiung City. J Chin Soc Vet Sci. 1999;25:241-5 (in Chinese).

10 Su YC. Epidemiological studies on zoonotic helminth and protozoan parasites in stray dogs and cats in middle-southern Taiwan. Chin J Parasitol. 1999;12:11-22

11 Fan CK, Tsai YY, Su KE, Lin JC. Epidemiological survey on zoonoses intestinal helminths and blood parasites among dogs in aboriginal villages of Bunun in Da-Wu and Dan-Zen districts, Taitung County. Chin J Parasitol. 2000;13:47-52 (in Chinese).

12 Fan CK, Su KE, Lin YH, Liao CW, Du WY, Chiou HY. Seroepidemiologic survey of Dirofilaria immitis infection among domestic dogs in Taipei City and mountain aboriginal districts in Taiwan (1998-1999). Vet Parasitol. 2001;102: 113-20.

13 Wu CC, Fan PC. Prevalence of canine dirofilariasis in Taiwan. J Helminthol. 2003;77:83-88

14 Yang SF, Lai CM, Liu Cl, Tsai HJ, Liang SL, Yang HL. Seroprevalence of feline heartworm infection in Taipei area. Taiwan Vet J. 2005;31:2.

15 Lin CH, Lo PY, Tsai HJ, Wang LC, Liaw BS, Hsieh OF, Chang YS, Tsai YC, Yu SC, Chueh LL. Dirofilaria immitis exposure status in client-owned cats with or without lower airway/lung-associated signs: case-control study in a canine heartworm-endemic area. J Feline Med Surg. 2015;19:153-7.

16 Chou CH, Yeh TM, Lu YP, Shih WL, Chang CD, Chien CH, Liu SS, Wu HY, Tsai FJ, Huang HH, Liao MH. Prevalence of zoonotic pathogens by molecular detection in stray dogs in Central Taiwan. Thai J Vet Med. 2014:44:3.

17 Song KH, Park JE, Lee DH, Lee SH, Shin HJ. Serological update and molecular characterization of Dirofilaria immitis in dogs, South Korea. Res Vet Sci. 2010;88:467-9.

18 Hou H, Shen G, Wu W, Gong P, Liu Q, You J, Cai Y, Li J, Zhang X. Prevalence of Dirofilaria immitis infection in dogs from Dandong, China. Vet Parasitol. 2011;183:1.

19 Oi M, Yoshikawa S, Ichikawa Y, Nakagaki K, Matsumoto J, Nogami S. Prevalence of Dirofilaria immitis among shelter dogs in Tokyo, Japan, after a decade: comparison of 1999-2001 and 2009-2011. Parasite. 2014:21:10.

20 Grieve RB, Lok JB, Glickman LT. Epidemiology of canine heartworm infection. Epidemiol Rev. 1983;5:1

21 Nelson CT, McCall JW, Carithers D. Current canine guidelines for the diagnosis, prevention, and Management of Heartworm (Dirofilaria immitis) infection in dogs, American heartworm society, 2014, https://www. heartwormsociety.org/

22 Marcos R, Pereira C, Santos M, Luzzago C, Lauzi S, Maia J, Faustino A, Puente-Payo P. Buffy coat smear or Knott's test: which to choose for canine microfilaria screening in field studies? Vet Clin Pathol. 2016;45:1.

23 Lee AC, Atkins CE. Understanding feline heartworm infection: disease, diagnosis, and treatment. Top Companion Anim Med. 2010;25:4.

24 Venco L, Marchesotti F, Manzocchi S. Feline heartworm disease: a 'Rubik's-cube-like'diagnostic and therapeutic challenge. J Vet Cardiol. 2015;17:S190-S201.

25 Atkins CE, DeFrancesco TC, Coats JR, Sidley JA, Keene BW. Heartworm infection in cats: 50 cases (1985-1997). J Am Vet Med Assoc. 2000;217:3.
26 Litster AL, Atwell RB. Feline heartworm disease: a clinical review. J Feline Med Surg. 2008;10:2

27 Little SE, Raymond MR, Thomas JE, Gruntmeir J, Hostetler JA, Meinkoth JH, Blagburn BL. Heat treatment prior to testing allows detection of antigen of Dirofilaria immitis in feline serum. Parasit Vectors. 2014;7:1.

28 Berdoulay P, Levy JK, Snyder PS, Pegelow MJ, Hooks JL, Tavares LM, Gibson NM, Salute ME. Comparison of serological tests for the detection of natural heartworm infection in cats. J Am Anim Hosp Assoc. 2004;40:5.

29 Snyder PS, Levy JK, Salute ME, Gorman SP, Kubilis PS, Smail PW, George LL. Performance of serologic tests used to detect heartworm infection in cats. J Am Vet Med Assoc. 2000;216:5.

30 Liu J, Song KH, Lee SE, Lee JY, Lee Jl, Hayasaki M, You MJ, Kim DH. Serological and molecular survey of Dirofilaria immitis infection in stray cats in Gyunggi province, South Korea. Vet Parasitol. 2005;130:1.

31 Roncalli RA, Yamane Y, Nagata T. Prevalence of Dirofilaria immitis in cats in Japan. Vet Parasitol. 1998;75:1.

32 Miller MW, Atkins CE, Stemme K, Robertson-Plouch C, Guerrero J. Prevalence of exposure to Dirofilaria immitis in cats in multiple areas of the United States. Vet Ther. 2000;1:3.

33 Kramer L, Genchi C. Feline heartworm infection: serological survey of asymptomatic cats living in northern Italy. Vet Parasitol. 2002;104:1.

34 Kalkstein TS, Kaiser L, Kaneene JB. Prevalence of heartworm infection in healthy cats in the lower peninsula of Michigan. J Am Vet Med Assoc. 2000; $217: 6$

\section{Submit your next manuscript to BioMed Central and we will help you at every step:}

- We accept pre-submission inquiries

- Our selector tool helps you to find the most relevant journal

- We provide round the clock customer support

- Convenient online submission

- Thorough peer review

- Inclusion in PubMed and all major indexing services

- Maximum visibility for your research

Submit your manuscript at www.biomedcentral.com/submit
Biomed Central 